\title{
Distributed Contention-Aware Call Admission Control for IEEE 802.11 Multi-Radio Multi-Rate Multi-Channel Wireless Mesh Networks
}

\author{
Tehuang Liu Wanjiun Liao \\ Department of Electrical Engineering \\ National Taiwan University \\ Taipei, Taiwan \\ \{tehuangliu,wjliao\}@ntu.edu.tw
}

\begin{abstract}
In this paper, we focus on call admission control (CAC) in IEEE 802.11 multi-radio multi-rate multi-channel $\left(\mathrm{MR}^{2}-\mathrm{MC}\right)$ wireless mesh networks (WMNs). CAC is the key component of QoS routing protocols. The goal of CAC is to protect existing flows from QoS violations and fully utilize available radio resource on channels. We propose a CAC mechanism, called Contention-Aware Multi-channel Call Admission Control (CMC), for $\mathrm{MR}^{2}$-MC WMNs based on IEEE 802.11 DCF. CMC is fully distributed, relies on local information to estimate the residual bandwidth of a path, and can be integrated into existing routing protocols for $\mathrm{MR}^{2}$-MC WMNs to provide QoS. We evaluate the performance of CMC via ns-2 simulations. The results show that CMC can precisely predict the end-to-end residual bandwidths of paths, successfully protects existing flows from QoS violations, and fully utilizes the bandwidths on channels.
\end{abstract}

\section{Categories and Subject Descriptors}

C.2.1 [Network Architecture and Design]: Wireless communication

\section{General Terms}

Design, Performance

\section{Keywords}

wireless mesh networks, call admission control, multi-radio, multi-rate, multi-channel

\section{INTRODUCTION}

Wireless mesh networks (WMNs) have received much attention in recent years thanks to such desirable features as

\footnotetext{
Permission to make digital or hard copies of all or part of this work for personal or classroom use is granted without fee provided that copies are not made or distributed for profit or commercial advantage and that copies bear this notice and the full citation on the first page. To copy otherwise, to republish, to post on servers or to redistribute to lists, requires prior specific permission and/or a fee.
}

Qshine08 July 28-31, 2008, Hong Kong, China Copyright 2008 ICST ISBN 978-963-9799-26-4 DOI 10.4108/ICST.QSHINE2008.3941 low up-front cost, ease of maintenance, robustness, and reliable service coverage [1-2]. In a WMN, each node plays both roles of a host and a router, and is typically stationary and not power-constrained [3]. Some of the nodes in the network may have directed connections to the wired networks, serving as gateways for the other nodes to access the Internet. Packets are forwarded in a multi-hop fashion to and from the gateway node or between two non-gateway nodes. In wireless multi-hop networks, the capacity degradation problem [4] due to the inherent problem of interference between wireless nodes is a crucial issue. Employing multiple non-overlapping channels [5-6] has been regarded as an effective approach to overcoming this problem in WMNs. However, this approach may require existing protocols and algorithms to be redesigned to take advantage of channel diversity to increase the capacity gain.

In this paper, we focus on call admission control (CAC) for bandwidth-constrained flows in multi-channel WMNs based on IEEE 802.11 DCF. CAC is the key component of QoS provisioning. Due to the contention nature of IEEE 802.11 DCF, QoS is a challenging problem, even in single-channel IEEE 802.11 WMNs. The crux of the problem is to accurately estimate the residual bandwidth of a path. If the residual bandwidth of a path is overestimated, too many flows may be admitted into the system, depriving existing flows of the reserved bandwidths. On the other hand, a conservative estimation may underestimate the residual bandwidth, which allows better protection for existing flows but may result in the degradation of channel utilization and system throughput. As indicated in [7-8], inter-flow and intra-flow interference affect the end-to-end residual bandwidth of a path. However, the end-to-end bandwidth calculation problem, even in single-channel TDMA-based wireless networks, is NP-hard [9]. Therefore, it is important to find an efficient heuristic for this problem. Yang and Kravet [10] show that the available bandwidth that a node can use without causing QoS violations to existing flows (which pass through nodes within its interference range) is jointly determined by all nodes within its carrier-sensing range, not just by this node itself. They then propose an admission control framework, called Contention-aware 
Admission Control Protocol (CACP), to support bandwidth-constrained routing in single-channel ad hoc networks. The problem is exacerbated by the multi-rate capability in IEEE 802.11 PHY layer, which enables nodes to communicate with each other at different data rates under different channel conditions. As a result, the inter-flow contention cannot be obtained from the aggregate of bandwidth requirements of nodes within the carrier-sensing range. Similarly, the links on a path may operate at different transmission rates, which means that the intra-flow contention cannot simply be estimated as a multiple of the single hop bandwidth requirement.

In this paper, we focus on bandwidth-constrained flows. Each node in the network is equipped with multiple radios and acts as a Transit Access Point (TAP) [3], i.e., it has its own locally generated data and relayed packets to send. Each node may communicate with different neighbors at different data rates using different modulation and coding schemes to combat channel deterioration. We propose a CAC mechanism, called Contention-aware Multi-channel Call Admission Control (CMC), for multi-radio multi-rate multi-channel $\left(\mathrm{MR}^{2}\right.$-MC) WMNs based on IEEE 802.11 DCF. CMC is fully distributed, relies only on local information to estimate the end-to-end residual bandwidth of a path, and can be integrated into any routing protocol for $\mathrm{MR}^{2}$-MC WMNs to enable QoS provisioning. Moreover, CMC is applicable to multi-channel WMNs with arbitrary static channel assignment algorithms [5, 11]. We evaluate the performance of CMC with ns-2 simulations. The results show that CMC can precisely predict the end-to-end residual bandwidths of paths, protects existing flows from QoS violations, and fully utilizes the bandwidths on channels. To our best knowledge, this is the first paper providing a call admission control scheme for IEEE $802.11 \mathrm{MR}^{2}$-MC WMNs.

The rest of the paper is organized as follows. Section 2 describes the system model considered in this paper. Section 3 elaborates on CMC. Section 4 presents the ns-2 simulation results. Finally, we conclude the paper in Section 5.

\section{SYSTEM MODEL}

We consider an $\mathrm{MR}^{2}$-MC WMN with the IEEE 802.11 DCF MAC protocol. Nodes in the network are all stationary and act as Transit Access Points (TAPs) [3]. Packets are forwarded via multi-hop relaying. Each node is equipped with multiple radios and the number of radios at each node may be different. The channel assignment for radios is given and static [5, 11] (i.e., each assignment lasts for a long duration). The call admission control scheme proposed in this paper is designed for general channel assignments and thus applicable to WMNs with arbitrary static channel assignment algorithms. Two nodes are said to be one-hop neighbors (or neighbors for short) on channel $k$ if they have a radio tuned to channel $k$ and fall within the transmission range of each other. The transmission range and the carrier-sensing range of each node are denoted by $r_{t}$ and $r_{i}$, respectively. The multi-rate capability in the PHY layer is also considered in our model. We consider two types of flows. The first is the flow between a non-gateway node and a gateway node (connected to the Internet). The second is the flow between two non-gateway nodes. All flows are dynamically generated, i.e., no prior knowledge of traffic demands is given. Each flow has its bandwidth requirement.

In a multi-channel multi-radio WMN, when a node needs to broadcast a control message to its neighbors for certain network management operations (e.g., routing [6], load-balancing channel assignment [5], topology control [12], and flow redirection [12]), it can simply duplicate such message to each associated channel for transmission. However, this approach is inefficient and may incur high control overhead. An alternative solution [13] is to let nodes periodically rendezvous on a common channel to exchange control messages, but this approach requires synchronization between nodes. Shi et al. [14] propose a channel coordination protocol for exchanging control messages between nodes in CSMA wireless networks without the reliance on synchronization. The idea is to let nodes that have no data packets to send or receive keep listening on a dedicated control channel. As a result, it may suffer the missing neighbor problem (or called the deafness problem) [14]. A simple method [5-6, 12] that generates lower message overhead, does not require synchronization between nodes, and avoids the deafness problem is to employ an extra radio tuned to a dedicated control channel permanently such that a node can broadcast control messages to its neighbors using this radio. In this paper, for simplicity, we adopt the last approach.

\section{CONTENTION-AWARE}

\section{MULTI-CHANNEL CALL ADMISSION}

\section{CONTROL (CMC) \\ 3.1 Channel Residual Bandwidth}

To determine if there is enough bandwidth available on a path for a requesting flow, we start by estimating the local channel residual bandwidth at each node in terms of how much air time on the channel is free-to-use, i.e., available for future transmissions. We let each node maintain a table, called the BusyPeriod table. Let BusyPeriod denote the $^{2}$ BusyPeriod table at node $i$. The entry with index $n$ in the BusyPeriod $_{i}$ table, denoted by BusyPeriod $i[n]$, specifies the aggregate length of busy periods on channel $n$ perceived by node $i$ during a predefined period $T_{m}$, where $T_{m}$ is referred to as the measurement period in this paper. Note that a busy period includes all self transmission or receiving periods on the link. In addition, the period reserved by the network allocation vector (NAV) and any perceived transmission activity (determined by carrier-sensing) are also included. We use the example shown in Figure 1 to illustrate the measurement. In this example, there are six busy periods 


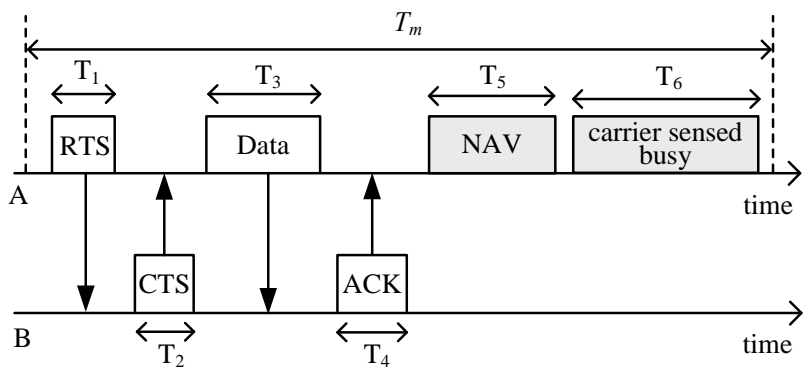

Figure 1. An example of measuring the busy periods.

during a $T_{m}$ period. A four-way RTS-CTS-Data-ACK handshake between nodes $A$ and $B$ causes four busy periods, i.e., $T_{1}, T_{2}, T_{3}$, and $T_{4}$, at node $A$. The fifth busy period, $T_{5}$, is the reserved period indicated by NAV at node $\mathrm{A}$. $\mathrm{T}_{6}$ means a busy period during which the carrier is sensed busy at node A. Note that $\mathrm{T}_{6}$ may be caused by the transmission activities at other nodes within the carrier-sensing range of node $\mathrm{A}$, or by environmental noise. Thus, the aggregate length of the busy periods at node $\mathrm{A}$ is the summation of these six busy periods.

Let BusyPeriod ${ }_{i}[n]_{m}$ denote the latest measurement of the aggregate length of busy periods on channel $n$ at node $i$. We update the historical value of $\operatorname{BusyPeriod}_{i}[n]$ by employing the exponential weighted averaging technique as follows.

$$
\begin{aligned}
& \text { BusyPeriod }_{i}[n] \\
& =\alpha \times \text { BusyPeriod }_{i}[n]+(1-\alpha) \times \text { BusyPeriod }_{i}[n]_{m},
\end{aligned}
$$

where $0 \leq \alpha \leq 1$. Note that, at the first measurement, the historical value is equal to the measurement.

Another table maintained at each node is called the ResidualAirTime table. Let ResidualAirTime $e_{i}$ denote this table at node $i$. The entry of index $n$ in the ResidualAirTime table, denoted by ResidualAirTime $i[n]$, indicates the estimate of the amount of free-to-use air time on channel $n$ at node $i$. IEEE 802.11 DCF uses carrier sensing to provide efficient collision avoidance. Thus, transmissions at one node may consume the available resource of all nodes within its carrier-sensing range. Therefore, to calculate the value of ResidualAirTime $_{i}[n]$, we let each node explicitly exchange the perceived channel status with the nodes within its carrier-sensing range. Specifically, we let each node broadcast its BusyPeriod table as a BUSY_PERIOD_REPORT message on the control channel to its carrier-sensing neighbors every $T_{b x}$ seconds, where $T_{b x}$ is referred to as the busy period exchange interval. Since the carrier-sensing range is often more than twice the transmission range, the exchange is performed via multi-hop forwarding. Without a position-locating system, we approximate the carrier-sensing neighborhood of a node, i.e., the set of nodes within its carrier-sensing range, as its $k$-hop neighborhood, where $k$ is a positive integer. Thus, we set the time-to-live (TTL) field in each BUSY_PERIOD_REPORT message to $k$, which results in a local broadcast. Each node also maintains a set of tables, called the NeighborBusyPeriod tables for each data NIC, to store the busy periods reported by its carrier-sensing neighbors. Let NeighborBusyPeriod ${ }_{i, n}$ denote the table for channel $n$ at node $i$. The entry of index $j$ in the NeighborBusyPeriod ${ }_{i, n}$ table, denoted by NeighborBusyPeriod $_{i, n}[j]$, represents the latest reported value of node $j$ 's busy periods on channel $n$. If a node does not receive any update on the busy period value of a carrier-sensing neighbor for $T_{n b}$ seconds, where $T_{n b}$ is the neighbor broadcast timeout, the entry for this neighbor in the NeighborBusyPeriod ${ }_{i, n}$ table will be removed.

According to the NeighborBusyPeriod tables, each node updates its ResidualAirTime table every $T_{c r}$ seconds, where $T_{c r}$ is referred to as the channel refresh interval. For node $i$, the new value of ResidualAirTime $i[n]$ is updated as follows.

$$
\begin{aligned}
& \text { ResidualAirTime }_{i}[n] \\
& =T_{m}-\max _{j \in\left\{i, E_{i, n}\right\}}\left(\text { NeighborBusyPeriod }_{i, n}[j]\right),
\end{aligned}
$$

where $E_{i, n}$ is the collection of nodes, each of which has an entry in the NeighborBusyPeriod ${ }_{i, n}$ table. Note that by definition, NeighborBusyPeriod ${ }_{i, n}[i]$ represents the busy periods observed by node $i$ itself on channel $n$, and thus is equivalent to BusyPeriod $i[n]$. Equation (2) follows because a node can interfere with any node within its carrier-sensing range and thus the maximal free-to-use channel air time at this node is determined by the carrier-sensing neighbor which perceives the busiest channel.

The accuracy of the estimate of the residual channel air time depends on the reporting rate of busy periods of the carrier-sensing neighbors, so employing a $T_{b x}$ may improve the accuracy of the estimation. However, a smaller $T_{b x}$ leads to higher control message overhead. Thus we propose a threshold-triggered approach for exchanging BUSY_PERIOD_REPORT messages between nodes and let $T_{b x}$ remain large. Specifically, we let each node remember its last reported busy periods and initiate the BUSY_PERIOD_REPORT message broadcasting procedure only when the difference between the current value and the last reported value is detected to be larger than the threshold $T S_{b p r}$, where $T S_{b p r}$ is referred to as the busy period reporting threshold. Note that if the BUSY_PERIOD_REPORT message is sent according to this threshold-based strategy, it needs only to contain the entries in the BusyPeriod table which exceed the threshold.

\subsection{Bandwidth Consumption}

The expected busy time (EBT) on a link is defined as the required air time for transmitting one packet over the link successfully. It can be considered the consumed bandwidth from the single-hop transmission perspective. Assume that the four-way RTS-CTS-Data-ACK handshake is adopted. 
The EBT of a successful transmission is given by

$$
E B T=T_{R T S}+T_{C T S}+\frac{L}{R}+T_{A C K},
$$

where $T_{R T S}, T_{C T S}, T_{A C K}$ are the air time needed to transmit the RTS, CTS, and ACK control frames, respectively, $L$ is the data frame size, and $R$ is the transmission rate on the link. If the packet loss rate (PLR) of data frames is considered, (3) is re-expressed by

$$
E B T=\frac{1}{1-P L R}\left(T_{R T S}+T_{C T S}+\frac{L}{R}+T_{A C K}\right) .
$$

Equation (4) follows because the expected number of Bernoulli trials to get the first success with parameter (1-PLR) is $1 /(1-P L R)$ [15]. Here, we ignore the additional channel busy time caused by the losses of RTS and CTS frames, since such losses are relatively rare. The packet loss rate can be approximated by using the broadcast packet technique described by De Couto et al. [16].

Based on EBT, we calculate the actual consumed bandwidth on a link for transmitting a packet along a path with the consideration of intra-flow contention. To illustrate the effect of intra-flow contention on the bandwidth consumption, we consider a five-hop routing path as shown in Figure 2. This path is composed of nodes A, B, C, D, E, and $\mathrm{F}$. The number associated with each link indicates the channel on which the link operates. The dotted line connecting links specifies their interference relationships. For example, link B-C interferes with links A-B and D-E but not with link C-D or link E-F. Let $E B T_{x-y, f}$ denote the expected busy time on the channel for successfully transmitting a packet of flow $f$ over link $x-y$. Now consider link D-E. For flow $f$, the total perceived channel busy time on link D-E for transmitting a packet from node $\mathrm{A}$ to node $\mathrm{F}$ equals $\left(E B T_{D-E, f}+E B T_{B-C, f}+E B T_{E-F, f}\right)$, where the last two terms, $E B T_{B-C, f}$ and $E B T_{E-F, f}$, account for the extra consumed air time on link D-E due to the intra-flow contention on links $\mathrm{B}-\mathrm{C}$ and $\mathrm{E}-\mathrm{F}$. This aggregate channel busy time is referred to as the cumulative EBT (CEBT) in this paper. For flow $f$, the CEBT of link $x-y$ on path $p$ (denoted by $C E B T_{x-y, p, f}$ ) can be calculated as

$$
C E B T_{x-y, p, f}=\sum_{i \in\left\{I_{x-y} \cap p\right\}} E B T_{i, f},
$$

where $p$ denotes the set of links on path $p$, and $I_{x-y}$ denotes the set of links within the carrier-sensing range of link $x-y$ and operating on the same channel as link $x-y$. Here we say that link $x-y$ interferes with link $u-v$ (or that link $u-v$ is within the interference range of link $x-y$ ), if at least one of nodes $u$ and $v$ is located within the union of the interference ranges of nodes $x$ and $y$.

$C E B T_{x-y, p, f}$ can be interpreted as "the total resource consumption on link $x-y$ " for transmitting one packet of flow

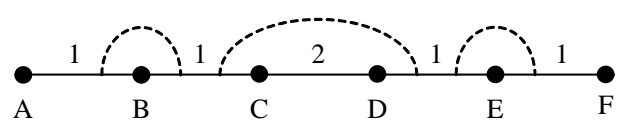

Figure 2. An example to illustrate the intra-flow contention.

$f$ along path $p$, where the resource means the "air time." However, without position-locating systems it is difficult to know whether a link interferes with another link or not. In this paper, we approximate the interfering links of a link as its $k$-hop neighboring links.

\subsection{Residual Path Capacity}

Before obtaining the residual capacity of a path, we first calculate the residual capacity of each link along this path. Consider a requesting flow, say $f$. Let $B_{f}$ and $L_{f}$ denote its bandwidth requirement and the data frame size, respectively. Let $R L C_{x-y, p, f}$ denote the residual capacity of link $x-y$ along path $p$ for flow $f$. Suppose that link $x-y$ operates on channel $n$. Then, $R L C_{x-y, p, f}$ is calculated as follows.

$$
R L C_{x-y, p, f}=\frac{\min _{i \in\{x, y\}}\left(\text { ResidualAirTime }_{i}[n]\right)-\beta \times T_{m}}{C E B T_{x-y, p} \times\left(B_{f} \times T_{m} \times \frac{1}{L_{f}}\right)},
$$

where $\beta$ is a tolerance factor and has a value between 0 and 1 , and the term $\left(B_{f} \times T_{m} \times 1 / L_{f}\right)$ represents the number of frames needed to be transmitted over the link during a $T_{m}$ period to satisfy the bandwidth requirement of flow $f$. The residual capacity of a link obtained from (6) can be viewed as the maximum number of flows (with the same requirements as flow $f$ ) which can be admitted on path $p$ from the perspective of link $x-y$.

The bottleneck of a path, i.e., the link with the smallest residual capacity on the path, determines the actual residual capacity of this path. Let $B R L C_{p, f}$ denote the bottleneck RLC (BRLC) of a path $p$ for flow $f$. We have

$$
B R L C_{p, f}=\min _{i \in p}\left(R L C_{i, p, f}\right) .
$$

Consider two paths $p$ and $q$. If $B R L C_{p}$ is smaller than $B R L C_{q}$, then the bottleneck of path $p$ is more congested than the bottleneck of path $q$. Since packets on path $q$ can be propagated more quickly and experience less contentions, path $q$ should be a better choice than path $p$. However, if path $q$ is much longer than path $p$, it is hard to say which one is better. Thus, we further take account of the factor of path length when estimating the residual capacity of a path, and rewrite (7) as (8).

$$
\text { normalized } \begin{aligned}
B R L C_{p, f} & \equiv N B R L C_{p, f} \\
& =\mu^{G} \times \min _{i \in p} R L C_{i, p, f},
\end{aligned}
$$


where $G$ is the length of path $p$ in hops, and $\mu$ is a tunable system parameter with a value between 0 and 1 . Equation (8) is interpreted as follows. A longer path may have more capability to steer clear of congestion areas and consequently larger capacity. However, a longer path implies that more resource is consumed and the end-to-end delay may also increase. In addition, a longer path also implies that the packet has more chances of being dropped at intermediate nodes due to the overflow at sending queues, or transmission failures. Thus, $\mu$ can be regarded as the average probability of not being dropped at each intermediate node.

\subsection{Integration into Routing Protocols}

CMC can be integrated into existing routing protocols for $\mathrm{MR}^{2}-\mathrm{MC} \mathrm{WMNs}$ to enable QoS routing. $\mathrm{CMC}$ is responsible for determining the feasibility of a discovered path for the routing agent. If the routing agent at the ingress node of the requesting flow cannot discover any feasible path, i.e., any path whose NBRLC is greater than or equal to one, this flow will be blocked. Note that (6) to (8) imply that a path is feasible if and only if all its sub-paths are feasible. In other words, the routing protocol will discard a candidate partial path if it fails in the feasibility test (since it leads to an infeasible path ultimately).

When a route is required by a new flow, the source node initiates the route discovery procedure by flooding a ROUTE REQUEST (RREQ) packet on the control channel. In addition to the profile of the requesting flow, the RREQ packet must carry the following information for intermediate nodes to compute the path capacity hop by hop.

- the channel map at the previous hop,

- the ResidualAirTime table at the previous hop,

- the number of entries in each of the

NeighborBusyPeriod tables at the previous hop, and

- the partial path information discovered so far.

The format of an $m$-hop partial path is expressed by

$$
\begin{aligned}
& \left\{\left(\mathrm{N}_{1}, \mathrm{C}_{1}, \mathrm{E}_{1}, \mathrm{CE}_{1}\right),\left(\mathrm{N}_{2}, \mathrm{C}_{2}, \mathrm{E}_{2}, \mathrm{CE}_{2}\right), \ldots,\right. \\
& \left.\left(\mathrm{N}_{m-1}, \mathrm{C}_{m-1}, \mathrm{E}_{m-1}, \mathrm{CE}_{m-1}\right),\left(\mathrm{N}_{m}, \mathrm{C}_{m}, \mathrm{E}_{m}, \mathrm{CE}_{m}\right)\right\} .
\end{aligned}
$$

The four fields, $\mathrm{N}_{i}, \mathrm{C}_{i}, \mathrm{E}_{i}$, and $\mathrm{CE}_{i}$, are defined as follows.

- $\mathrm{N}_{i}, i=1$ to $m$, is the identification of the $i^{\text {th }}$ relaying node.

- $\quad \mathrm{C}_{i}, i=1$ to $m$, specifies the channel used at the $i^{\text {th }}$ hop.

- $\quad \mathrm{E}_{i}, i=1$ to $m$, is the value of $E B T$ on the link at the $i^{\text {th }}$ hop, which is calculated according to (4).

- $\quad \mathrm{CE}_{i}, i=1$ to $m$, is the value of $C E B T$ on the link at the $i^{\text {th }}$ hop, which shall be updated according to (5) when a new node is attached to the partial path.

When a node receives an RREQ packet, if there is no common channel (excluding the control channel) used by itself and the previous hop node, which forwards this RREQ packet to it, this RREQ packet is dropped. Otherwise, the routing agent forms a new partial path by appending one new link (i.e., a link between the node and the previous hop node) to the end of the original partial path. Note that the routing agent needs to decide the channel on which this newly added link operates according to the implemented routing algorithm. Once the new partial path is determined, the routing agent uses (8) to calculate NBRLC to examine whether this new partial path meets the bandwidth requirement of the flow. If it finds that this new partial path is not feasible, the RREQ packet is discarded. Another possible strategy is that when the routing agent finds that the resulting new partial path fails in the feasibility test, it selects another common channel for the newly added link to result in another candidate partial path and only when there is no feasible candidate partial path will it discard this RREQ packet.

If the new partial path passes the feasibility test, the node updates the RREQ packet by attaching this channel, its identification, and the corresponding $E_{i}$ and $C E_{i}$ values to the partial path field. In addition, it updates the previous $C E$ fields in the partial path according to (5) if necessary (due to the extra intra-flow contention caused by the newly added link in the partial path). If this node is not the destination, it then rebroadcasts this RREQ packet on the control channel. Otherwise, it unicasts a ROUTE REPLY (RREP) packet back to the source. The resulting path is stored in the RREQ packet. Each intermediate node receiving an RREP packet knows the channels used to communicate to the previous hop (downstream) and next hop (upstream) nodes. It then establishes the forward and reverse paths accordingly.

\section{PERFORMANCE EVALUATION}

\subsection{Simulation Model}

We study the performance of CMC via ns-2 simulations [17]. We divide a $1170 \mathrm{~m} \times 1170 \mathrm{~m}$ area into $9 \times 9$ square cells as shown in Figure 3 . We place one node in the center of each cell, so there are 81 nodes in the network. Each node has a radio propagation range of 225 meters and a carrier-sensing range of 450 meters. The number of physical channels is 12, including 11 data channels and one dedicated control channel. Each node is equipped with four data NICs and one control NIC tuned to the control channel. We adopt a general channel assignment strategy, called random channel assignment strategy [6, 18], where the four data NICs at each node are randomly assigned four different data channels. However, this strategy may result in orphan NICs, i.e., an NIC at a node may be assigned a channel which is not common to any channel used by the neighboring nodes. An orphan NIC is useless since no neighboring nodes can receive its data, but it enables us to simulate the condition in which nodes have different numbers of effective radios (i.e., non-orphan radios). Specifically, if an assignment leads to a node with $x$ orphan NICs, it means this node is equipped with 


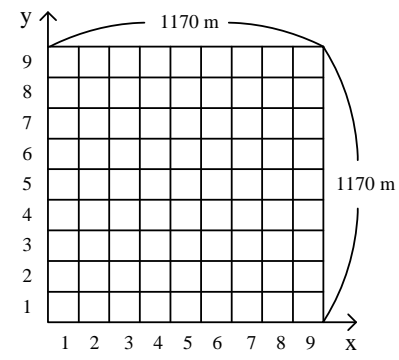

Figure 5. A $1170 \mathrm{~m} \times 1170 \mathrm{~m}$ area divided into $9 \times 9$ square cells.

Table 1. Protocol parameter settings

\begin{tabular}{|c|c|c|c|c|c|}
\hline$r_{t}$ & $r_{i}$ & $T_{m}$ & $\alpha$ & $T_{b x}$ & $k$ \\
\hline 225 & 450 & $0.1 \mathrm{~s}$ & 0.5 & $1.0 \mathrm{~s}$ & 3 \\
\hline \hline$T_{n b}$ & $T_{c r}$ & $T S_{b p}$ & $\beta$ & $\mu$ & $R$ \\
\hline $2.0 \mathrm{~s}$ & $0.1 \mathrm{~s}$ & $1 \mathrm{~ms}$ & 0.25 & 0.95 & 3 \\
\hline
\end{tabular}

only 4- $x$ effective data NICs and one control NIC, where $0 \leq$ $x<4$. Note that we do not allow a node with no effective data NIC. The data rate on each link between any two neighboring nodes is randomly chosen from $6,9,12,18,24$, 36, 48, and 54 Mbps, which are supported by IEEE 802.11a [19]. The error rate of data packets on each link is randomly chosen from $0.1 \%, 0.5 \%, 1 \%, 5 \%$, and $10 \%$. The other parameter settings of CMC are listed in Table 1.

In the simulation, CMC is integrated into Capacity-Aware Routing (CAR) [6] to enable QoS, where CAR is an on-demand load-balancing routing protocol for $\mathrm{MR}^{2}$-MC WMNs and designed for best-effort data flows. In [6], CAR is shown to be able to fully utilize the network resource by distributing traffic across channels and among nodes. Two scenarios are considered in the simulation. The first is the $\mathrm{ad}$ hoc mode, in which one CBR flow is generated for two randomly selected source and destination nodes every two seconds. The second is the wireless backhaul mode, in which the nodes in cells with coordinates of $(5,1)$ and $(5,9)$ are designated as the gateway nodes and one CBR flow destined to the Internet is generated at a randomly selected non-gateway node every two seconds. In both scenarios, the bandwidth requirement of each flow is $1.5 \mathrm{Mbps}$ and the data payload of each packet is 1000 bytes. The simulation time is 80 seconds.

\subsection{Simulation Results}

Figures 4 and 5 show the average flow delivery ratio in the ad hoc mode and the wireless backhaul mode, respectively. Clearly, in both modes, CMC guarantees about a $100 \%$ delivery ratio over the whole simulation period. Contrarily, when CMC is de-activated, the delivery ratio degrades severely as more flows enter the system. This proves that CMC can effectively protect existing flows from QoS violations. Figures 6 and 7 depict the system throughputs for

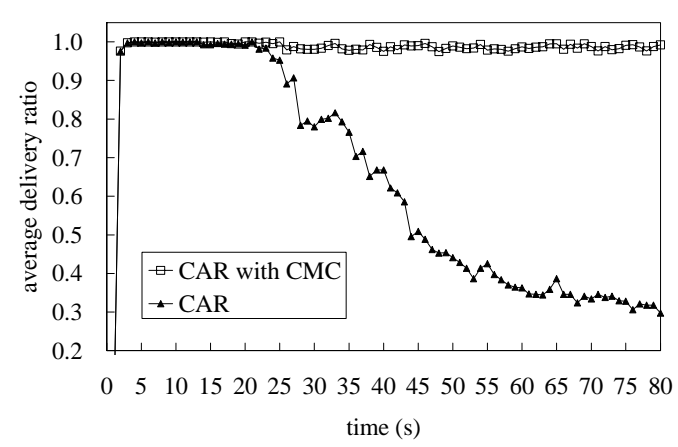

Figure 4. Average delivery ratio in the ad hoc mode.

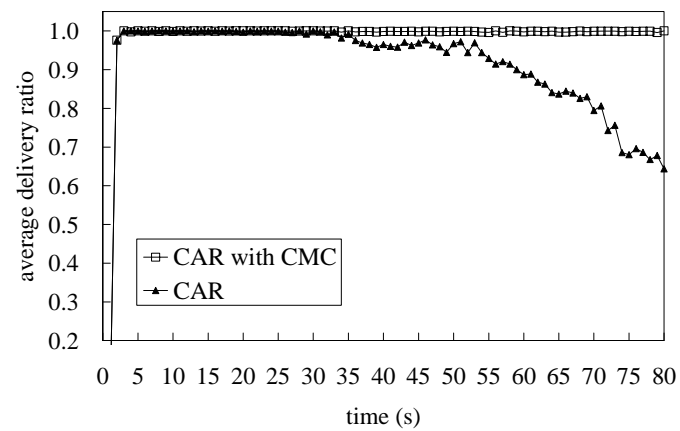

Figure 5. Average delivery ratio in the backhaul mode.

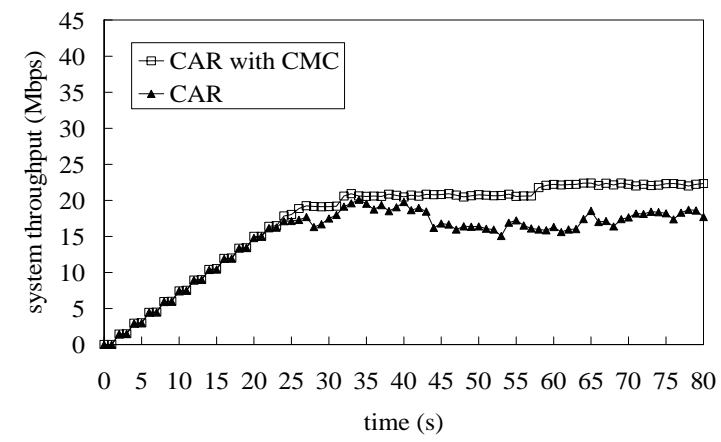

Figure 6. System throughput in the ad hoc mode.

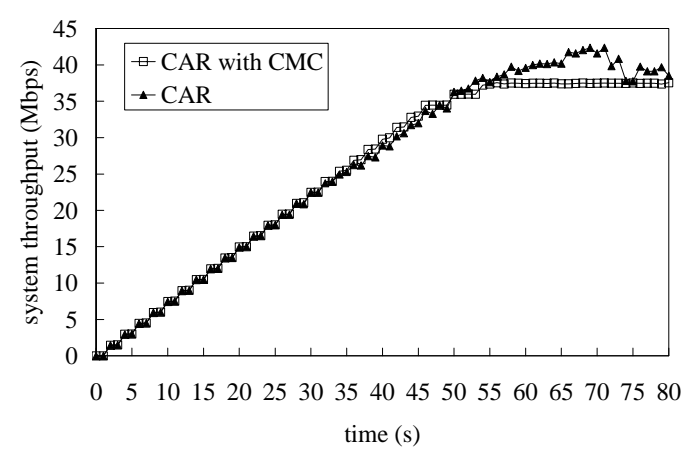

Figure 7. System throughput in the backhaul mode. 
the ad hoc and wireless backhaul modes, respectively. The system throughput is defined as the sum of the throughputs of all flows in the network. From these two figures, we find that CMC does not impact the maximum achieved system throughput very much, as compared with the system throughput achieved by CAR without CMC. This means that CMC is indeed efficient and does not excessively block new flows to protect existing flows. In fact, sometimes, CMC may even improve the system throughput. This is because if call admission control is de-activated, nodes may suffer from high probabilities of packet collisions and buffer overflow, especially when there are too many flows in the network, contending with each other.

\section{CONCLUSIONS}

In this paper, we propose a mechanism called Contention-Aware Multi-channel Call Admission Control (CMC) for $\mathrm{MR}^{2}-\mathrm{MC}$ WMNs based on IEEE 802.11 DCF. $\mathrm{CMC}$ is fully distributed, relies only on local information to estimate the end-to-end residual bandwidth of a path, and can be integrated into existing routing protocols for $\mathrm{MR}^{2}$-MC WMNs to enable QoS routing. The simulation results show that $\mathrm{CMC}$ is effective and efficient. It can precisely predict the end-to-end residual bandwidths of paths, successfully protects existing flows from QoS violations, and fully utilizes the bandwidths on channels. To our best knowledge, this is the first paper providing a call admission control scheme for IEEE 802.11 $\mathrm{MR}^{2}$-MC WMNs.

\section{ACKNOWLEDGEMENT}

This work was supported in part by National Science Council (NSC), Taiwan, under a Center Excellence Grant NSC95-2752-E-002-006-PAE, and in part by NSC under Grant Number NSC96-2628-E-002-003-MY3.

\section{REFERENCES}

[1] I. F. Akyildiz, X. Wang, and W. Wang. Wireless mesh networks: a survey. Computer Networks Journal (Elsevier), Vol. 47, No. 4, pp. 445-487, Mar. 2005.

[2] R. Bruno, M. Conti, and E. Gregori. Mesh Networks: Commodity Multihop Ad Hoc Networks. IEEE Comm., Vol. 43, Issue 3, pp. 123-131, Mar. 2005.

[3] V. Gambiroza, B. Sadeghi, and E. W. Knightly. End-to-End Performance and Fairness in Multihop Wireless Backhaul Networks. ACM MOBICOM, Sep.-Oct. 2004.

[4] A. E. Gamal, J. Mammen, B. Prabhakar, and D. Shah. Throughput-Delay Trade-off in Wireless Networks. IEEE INFOCOM, Mar. 2004.

[5] A. Raniwala and T.-C. Chiueh. Architecture and Algorithms for an IEEE 802.11-Based Multi-Channel WMN. IEEE INFOCOM, Mar. 2005.
[6] T. Liu and W. Liao. Capacity-Aware Routing in Multi-Channel Multi-Rate WMNs. IEEE ICC, Jun. 2006.

[7] J. Jun and M.L. Sichitiu. The Nominal Capacity of Wireless Mesh Networks. IEEE Wireless Communications, Vol. 10, Issue 5, pp. 8-14, Oct. 2003.

[8] K. Jain, J. Padhye, V. N. Padmanabhan, and L. Qiu. Impact of Interference on Multi-hop Wireless Network Performance. ACM MOBICOM, Sep. 2003.

[9] C. Zhu and M. S. Corson. QoS Routing for Mobile Ad Hoc Networks. IEEE INFOCOM, Jun. 2002.

[10] Y. Yang AND R. Kravets. Contention-Aware Admission Control for Ad Hoc Networks. IEEE Trans. on Mobile Computing, Vol. 4, Issue 4, pp. 363-377, July-Aug. 2005.

[11] A. K. Das, H. M. K. Alazemi, R. Vijayakumar, and S. Roy. Optimization Models for Fixed Channel Assignment in WMNs with Multiple Radios. IEEE SECON, Sep. 2005.

[12] K. N. Ramachandran, E. M. Belding, K. C. Almeroth, and M. M. Buddhikot. Interference-Aware Channel Assignment in Multi-Radio WMNs. IEEE INFOCOM, Apr. 2006.

[13] J. So and N. Vaidya. Multi-Channel MAC for Ad Hoc Networks: Handling Multi-Channel Hidden Terminals Using A Single Transceiver. ACM MOBIHOC, May 2004.

[14] J. Shi, T. Salonidis, and E. Knightly. Starvation Mitigation through Multi-Channel Coordination in CSMA based Wireless Networks. ACM MOBIHOC, May 2006.

[15] S. Ghahramani. Fundamentals of Probability with Stochastic Processes. Prentice Hall, 3rd edition, pp. 215-216, 2005.

[16] D. S. J. D. Couto, D. Aguayo, J. Bicket, and R. Morris. High-throughput path metric for multi-hop wireless routing. ACM MOBICOM, Sep. 2003.

[17] The Network Simulator - ns2. http://www.isi.edu/nsnam/ns/.

[18] T. Liu and W. Liao. On Routing in Multichannel Wireless Mesh Networks: Challenges and Solutions. IEEE Network, Vol. 22, No. 1, pp. 13-18, Jan.-Feb. 2008.

[19] IEEE 802.11. Part11: Wireless LAN Medium Access Control (MAC) and Physical Layer (PHY) Specifications: High-speed Physical Layer in the $5 \mathrm{GHz}$ Band. Supplement to IEEE 802.11 Standard, 1999. 